\title{
General Solution and Stability of Additive-Quadratic Functional Equation in IRN-Space
}

\author{
K. Tamilvanan $\mathbb{D}^{1},{ }^{1}$ Nazek Alessa $\mathbb{D}^{2}{ }^{2}$ K. Loganathan $\mathbb{D}^{\mathbb{D}},{ }^{3}$ G. Balasubramanian, ${ }^{1}$ \\ and Ngawang Namgyel $\mathbb{1}^{4}$ \\ ${ }^{1}$ Department of Mathematics, Government Arts College for Men, Krishnagiri, 635001 Tamil Nadu, India \\ ${ }^{2}$ Department of Mathematical Sciences, Faculty of Science, Princess Nourah Bint Abdulrahman University, Saudi Arabia \\ ${ }^{3}$ Research and Development Wing, Live4Research, Tiruppur, 638106 Tamil Nadu, India \\ ${ }^{4}$ Department of Humanities and Management, Jigme Namgyel Engineering College, Royal University of Bhutan, Dewathang, Bhutan
}

Correspondence should be addressed to K. Tamilvanan; tamiltamilk7@gmail.com

and Ngawang Namgyel; ngawangnamgyel@jnec.edu.bt

Received 20 June 2021; Revised 6 July 2021; Accepted 16 July 2021; Published 4 August 2021

Academic Editor: Liliana Guran

Copyright $\odot 2021 \mathrm{~K}$. Tamilvanan et al. This is an open access article distributed under the Creative Commons Attribution License, which permits unrestricted use, distribution, and reproduction in any medium, provided the original work is properly cited.

The investigation of the stabilities of various types of equations is an interesting and evolving research area in the field of mathematical analysis. Recently, there are many research papers published on this topic, especially additive, quadratic, cubic, and mixed type functional equations. We propose a new functional equation in this study which is quite different from the functional equations already dealt in the literature. The main feature of the equation dealt in this study is that it has three different solutions, namely, additive, quadratic, and mixed type functions. We also prove that the stability results hold good for this equation in intuitionistic random normed space (briefly, IRN-space).

\section{Introduction}

The theory of random normed spaces (RN-spaces) is important as a generalization of the deterministic result of linear normed spaces and also in the study of random operator equations. The RN-spaces may also provide us with the tools to study the geometry of nuclear physics and have important applications in quantum particle physics.

The concept of stability of a functional equation arises when one replaces a functional equation by an inequality which acts as a perturbation of the equation. The first stability problem concerning group homomorphisms was raised by Ulam [1] in 1940 and affirmatively solved by Hyers [2]. Aoki generalized the result of Hyers [3] for approximate additive mappings and by Rassias [4] for approximate linear mappings by allowing the difference Cauchy equation $\| f(x$ $+y)-f(x)-f(y) \|$ to be controlled by $\varepsilon\left(\|x\|^{p}+\|y\|^{p}\right)$. In 1994, a generalization of the Th.M. Rassias' theorem was got by Gavruta [5], who replaced $\varepsilon\left(\|x\|^{p}+\|y\|^{p}\right)$ by a general control function $\varphi(x, y)$. For additional information regard- ing the outcomes about such issues, the related background in [6-12] can be examined. Absorbing new outcomes concerning mixed-type functional equations has as of late been acquired by Najati et al. [13-15], Jun and Kim [16, 17], and Park [18-22].

The functional equations

$$
f(x+y)=f(x)+f(y)
$$

and

$$
f(x+y)+f(x-y)=2 f(x)+2 f(y),
$$

are called the additive and quadratic functional equations, respectively. Every solution of the additive and quadratic functional equations is said to be additive mapping and quadratic mapping, respectively.

As of late, Zhang [23] examined the cubic functional equation in intuitionistic random space. The stability of various equations in $\mathrm{RN}$-spaces has been as of late concentrated 
in Alsina [24], Eshaghi Gordji et al. [25, 26], Mihet and Radu [27-29], and Saadati et al. [30]. Xu et al. [31-33] presented the various mixed types of functional equations investigated in Intuitionistic fuzzy normed spaces, quasi Banach spaces, and random normed spaces. Also, Shu et al. [33-35] discussed various differential equations to study the HyersUlam stability, which provides a wide view of this stability problem.

In this present work, we introduce a new mixed type additive-quadratic functional equation

$$
\begin{aligned}
\varphi\left(\sum_{i=1}^{m} a^{i} v_{i}\right)+\sum_{i=1}^{m} \varphi\left(-a^{i} v_{i}+\sum_{j=1 ; i \neq j}^{m} a^{j} v_{j}\right) \\
=(m-3) \sum_{i=1}^{m} \varphi\left(a^{i} v_{i}+a^{j} v_{j}\right)-\left(m^{2}-5 m+2\right) \\
\quad \cdot \sum_{i=1}^{m} a^{2 i}\left[\frac{\varphi\left(v_{i}\right)+\varphi\left(-v_{i}\right)}{2}\right]-\left(m^{2}-5 m+4\right) \\
\\
\quad \cdot \sum_{i=1}^{m} a^{i}\left[\frac{\varphi\left(v_{i}\right)-\varphi\left(-v_{i}\right)}{2}\right],
\end{aligned}
$$

where $a$ is a fixed integer and $m \geq 5$ and investigate the UlamHyers stability results of this mixed type additive-quadratic functional equation in an intuitionistic random normed space.

So far various forms of additive and quadratic functional equations are considered in this research field to obtain their stability results through different methods. For the first time, a new mixed additive-quadratic functional equation is proposed in this paper, and its stability results are proved in an intuitionistic random normed space.

This type of functional equation can be of use in solving many physical problems and also has significant relevance in various scientific fields of research and study. In particular, additive-quadratic functional equations have applications in electric circuit theory, physics, and relations connecting the harmonic mean and arithmetic mean of several values. Providing a wealth of essential insights and new concepts in the field of functional equations.

\section{Preliminaries}

We recall the following ideas and conceptions of IRN-spaces in [36-41].

Definition 1 (see [42]). A mapping $\mu: \mathbb{R} \longrightarrow[0,1]$ is said to be a measure distribution function, if $\mu$ is left continuous on $\mathbb{R}$, non-decreasing, $\inf _{t \in \mathbb{R}} \mu(t)=0$, and $\sup _{t \in \mathbb{R}} \mu(t)=1$.

Definition 2 (see [42]). A mapping $v: \mathbb{R} \longrightarrow[0,1]$ is said to be a non-measure distribution function, if $v$ is right continuous on $\mathbb{R}$, non-increasing, $\sup _{t \in \mathbb{R}} v(t)=1$, and inf ${ }_{t \in \mathbb{R}} v(t)=0$.
Lemma 3 (see $[43,44])$. Let $L^{*}$ be a set with an operator $\leq_{L^{*}}$ is defined by

$$
\begin{gathered}
L^{*}=\left\{\left(v_{1}, v_{2}\right):\left(v_{1}, v_{2}\right) \in[0,1]^{2} \text { and } v_{1}+v_{2} \leq 1\right\}, \\
\left(v_{1}, v_{2}\right) \leq_{L^{*}}\left(w_{1}, w_{2}\right) \Leftrightarrow v_{1} \leq w_{1}, v_{2} \geq w_{2},\left(v_{1}, v_{2}\right),\left(w_{1}, w_{2}\right) \in L^{*} .
\end{gathered}
$$

Then, the pair $\left(L^{*}, \leq_{L^{*}}\right)$ is a complete lattice.

We denote its units by $0_{L^{*}}=(0,1)$ and $1_{L^{*}}=(1,0)$. Typically, a triangular norm (t-norm) $*=\Phi$ on $[0,1]$ is defined as an increasing, commutative, associative mapping $\Phi:[0,1]^{2} \longrightarrow[0,1]$ satisfying $\Phi(1, p)=1 * p=p$ for every $p \in[0,1]$, and a triangular conorm (t-conorm) $Y=\diamond$ is defined as an increasing, commutative, associative mapping $Y:[0,1]^{2} \longrightarrow[0,1]$ satisfying $Y(0, p)=0 \diamond p=p$ for all $p \in[0,1]$.

By using the lattice $\left(L^{*}, \leq_{L^{*}}\right)$, these definitions can be straightforwardly extended.

Definition 4 (see [44]). A triangular norm (t-norm) on $L^{*}$ is a mapping $\Phi:\left(L^{*}\right)^{2} \longrightarrow L^{*}$ satisfying the following conditions:

(i) Boundary condition

i.e., $\Phi\left(p, 1_{L^{*}}\right)=p, \forall p \in L^{*}$;

(ii) Commutativity

i.e., $\Phi(p, q)=\Phi(q, p), \forall(p, q) \in\left(L^{*}\right)^{2}$;

(iii) Associativity

i.e., $\Phi(p, \Phi(q, r))=\Phi(\Phi(p, q), r), \forall(p, q, r) \in\left(L^{*}\right)^{3}$;

(iv) Monotonicity

i.e., $p \leq_{L^{*}} p^{\prime}$ and $q \leq_{L^{*}} q^{\prime} \Rightarrow \Phi(p, q) \leq_{L^{*}} \Phi\left(p^{\prime}, q^{\prime}\right)$ for all $(p$, $\left.p^{\prime}, q, q^{\prime}\right) \in\left(L^{*}\right)^{4}$

If $\left(L^{*}, \leq_{L^{*}}, \Phi\right)$ is an Abelian topological monoid with unit $1_{L^{*}}$, then $\Phi$ is called a continuous t-norm.

Definition 5 (see [42]). A negator on $L^{*}$ is any decreasing mapping $N$ from $L^{*}$ to $L^{*}$ satisfying $N\left(1_{L^{*}}\right)=0_{L^{*}}$ and $N\left(1_{L^{*}}\right)=0_{L^{*}}$ . If $N(N(p))=p$ for all $p \in L^{*}$, then $N$ is called an involutive negator. A negator on $[0,1]$ is a decreasing mapping $N:[0,1] \longrightarrow[0,1]$ satisfying $N(0)=1$ and $N(1)=0$.

$N_{s}$ denotes the standard negator on $[0,1]$ defined by

$$
N_{s}(p)=1-p \text {, }
$$

for all $p \in[0,1]$.

Definition 6 (see [23]). Let $\mu$ and $\nu$ be measure and nonmeasure distribution functions from $V \times(0,+\infty)$ to $[0,1]$ such that

$$
\mu_{p}(t)+v_{p}(t) \leq 1, p \in V, t>0
$$


The triple $\left(V, I_{\mu, v}, \Phi\right)$ is said to be an intuitionistic random normed space if a vector space $V$, continuous trepresentable $\Phi$, and a mapping $I_{\mu, v}: V \times(0,+\infty) \longrightarrow L^{*}$ holds the following conditions: for all $p, q \in V$ and $t_{1}, t_{2}>0$

$$
\begin{gathered}
I_{\mu, v}(p, 0)=0_{L^{*}}, \\
I_{\mu, v}\left(p, t_{1}\right)=1_{L^{*}} \Leftrightarrow p=0, \\
I_{\mu, v}\left(\alpha p, t_{1}\right)=I_{\mu, v}\left(p, \frac{t_{1}}{|\alpha|}\right), \forall \alpha \neq 0, \\
I_{\mu, v}\left(p+q, t_{1}+t_{2}\right) \geq_{L^{*}} \Phi\left(I_{\mu, v}\left(p, t_{1}\right), I_{\mu, v}\left(q, t_{2}\right)\right) .
\end{gathered}
$$

Thus, $I_{\mu, \nu}$ is called an intuitionistic random norm. Hence,

$$
I_{\mu, v}\left(p, t_{1}\right)=\left(\mu_{p}\left(t_{1}\right), v_{p}\left(t_{1}\right)\right) .
$$

Example 1 (see [42]). Let $(V,\|\cdot\|)$ be a normed space. Let $\Phi(p, q)=\left(p_{1} q_{1}, \min \left(p_{2}+q_{2}, 1\right)\right)$ for all $p=\left(p_{1}, p_{2}\right), q=\left(q_{1}\right.$, $\left.q_{2}\right) \in L^{*}$ and let $\mu, v$ be measure and non-measure distribution functions defined by

$$
I_{\mu, v}(v, \varepsilon)=\left(\mu_{v}(\varepsilon), v_{v}(\varepsilon)\right)=\left(\frac{\varepsilon}{\varepsilon+\|v\|}, \frac{\|v\|}{\varepsilon+\|v\|}\right), \forall \varepsilon \in R^{+} .
$$

Then, $\left(V, I_{\mu, \nu}, \Phi\right)$ is an IRN-space.

Definition 7 (see [42]). Let $\left(V, I_{\mu, v}, \Phi\right)$ be an IRN-space.

(i) A sequence $\left\{p_{m}\right\}$ in $\left(V, I_{\mu, v}, \Phi\right)$ is known as a Cauchy sequence if, for some $\delta>0$ and $t>0$, there is an $m_{0}$ $\in \mathbb{N}$ satisfies

$$
I_{\mu, v}\left(p_{m}-p_{n}, t\right) \geq_{L^{*}}\left(N_{s}(\delta), \delta\right), m, n \geq m_{0} .
$$

(ii) The sequence $\left\{p_{m}\right\}$ is convergent to any point $p \in V$ if $I_{\mu, v}\left(p_{m}-p, t\right) \longrightarrow 1_{L^{*}}$ as $m \longrightarrow \infty$ for all $t>0$

(iii) An intuitionistic random normed space $\left(V, I_{\mu, v}, \Phi\right)$ is known as complete if every Cauchy sequence in $V$ is convergent to a point $p \in V$

\section{Solution of the Functional Equation (3)}

In this section, let us consider $V$ and $W$ are two real vector spaces.

Theorem 8. If an odd mapping $\varphi: V \longrightarrow W$ satisfies the functional equation (3) for all $v_{1}, v_{2}, \cdots, v_{m} \in V$, then the function $\varphi$ is additive.

Proof. In the view of the oddness of $\varphi$, we have $\varphi(-v)=-\varphi(v)$ for all $v \in V$. Using the oddness property, the functional equation (3) reduces as

$$
\begin{aligned}
& \varphi\left(\sum_{i=1}^{m} a^{i} v_{i}\right)+\sum_{i=1}^{m} \varphi\left(-a^{i} v_{i}+\sum_{j=1 ; i \neq j}^{m} a^{j} v_{j}\right) \\
& =(m-3) \sum_{i=1}^{m} \varphi\left(a^{i} v_{i}+a^{j} v_{j}\right)-\left(m^{2}-5 m+4\right) \sum_{i=1}^{m} a^{i} \varphi\left(v_{i}\right),
\end{aligned}
$$

for all $v_{1}, v_{2}, \cdots, v_{m} \in V$. Now, replacing $\left(v_{1}, v_{2}, \cdots, v_{m}\right)$ by $(0,0 \cdots, 0)$ in $(11)$, we get $\varphi(0)=0$. Interchanging $\left(v_{1}, v_{2}\right.$, $\left.\cdots, v_{m}\right)$ with $(v, 0,0, \cdots, 0)$ in $(11)$, we get

$$
\varphi(a v)=a \varphi(v), v \in V .
$$

Again interchanging $v$ with $a v$ in (12), we have

$$
\varphi\left(a^{2} v\right)=a^{2} \varphi(v),
$$

for all $v \in V$. Replacing $v$ by $a v$ in (13), we obtain

$$
\varphi\left(a^{3} v\right)=a^{3} \varphi(v), v \in V .
$$

From the equalities (12)-(14), we can generalize the results for any nonnegative integer $m$ as

$$
\varphi\left(a^{m} v\right)=a^{m} \varphi(v), v \in \mathrm{V} .
$$

Similarly, we have

$$
\varphi\left(\frac{v}{a^{m}}\right)=\frac{1}{a^{m}} \varphi(v), v \in V .
$$

Replacing $\left(v_{1}, v_{2}, \cdots, v_{m}\right)$ by $\left((x / a),\left(y / a^{2}\right), 0, \cdots, 0\right)$ in (11), we have

$$
\varphi(x+y)=\varphi(x)+\varphi(y), x, y \in V .
$$

Hence, the function $\varphi$ is additive.

Theorem 9. If an even mapping $\varphi: V \longrightarrow W$ satisfies the functional equation (3) for all $v_{1}, v_{2}, \cdots, v_{m} \in V$, then the function $\varphi$ is quadratic.

Proof. Since, in the view of evenness of $\varphi$, we have $\varphi(-v)=$ $\varphi(v)$ for all $v \in V$. Now, the functional equation (3) reduces as

$$
\begin{aligned}
& \varphi\left(\sum_{i=1}^{m} a^{\mathrm{i}} v_{i}\right)+\sum_{i=1}^{m} \varphi\left(-a^{i} v_{i}+\sum_{j=1 ; i \neq j}^{m} a^{j} v_{j}\right) \\
& =(m-3) \sum_{i=1}^{m} \varphi\left(a^{i} v_{i}+a^{j} v_{j}\right)-\left(m^{2}-5 m+2\right) \sum_{i=1}^{m} a^{2 i} \varphi\left(v_{i}\right) .
\end{aligned}
$$

for all $v_{1}, v_{2}, \cdots, v_{m} \in V$. Now, replacing $\left(v_{1}, v_{2}, \cdots, v_{m}\right)$ by $(0,0, \cdots, 0)$ in $(18)$, we obtain $\varphi(0)=0$. Interchanging $\left(v_{1}\right.$, $\left.v_{2}, \cdots, v_{m}\right)$ with $(v, 0,0, \cdots, 0)$ in $(18)$, we obtain

$$
\varphi(a v)=a^{2} \varphi(v), v \in V .
$$


Replacing $v$ by $a v$ in (19), we reach

$$
\varphi\left(a^{2} v\right)=a^{4} \varphi(v), v \in V
$$

Switching $v$ by $a v$ in (20), we get

$$
\varphi\left(a^{3} v\right)=a^{6} \varphi(v), v \in V
$$

From (19)-(21), we can generalize the results for any nonnegative integer $m$ as

$$
\varphi\left(a^{m} v\right)=a^{2 m} \varphi(v), v \in V
$$

Similarly, we have

$$
\varphi\left(\frac{v}{a^{m}}\right)=\frac{1}{a^{2 m}} \varphi(v), v \in V
$$

Replacing $\left(v_{1}, v_{2}, \cdots, v_{m}\right)$ by $\left((x / a),\left(y / a^{2}\right), 0, \cdots, 0\right)$ in (18), we obtain

$$
\varphi(x+y)+\varphi(x-y)=2 \varphi(x)+2 \varphi(y), x, y \in V
$$

Hence, the function $\varphi$ is quadratic.

Theorem 10. If a mapping $\varphi: V \longrightarrow W$ satisfies $\varphi(0)=0$ and satisfies the functional equation (3) for all $v_{1}, v_{2}, \cdots, v_{m} \in V$ if and only if there exists a symmetric biadditive mapping $Q: V \times V \longrightarrow W$ and $a$ additive mapping $A: V \longrightarrow W$ satisfies $\varphi(v)=Q(v, v)+A(v)$ for all $v \in V$.

Proof. Let a mapping $\varphi: V \longrightarrow W$ with $\varphi(0)=0$ satisfies the functional equation (3). We divide the function $\varphi$ into the odd part and even part as

$$
\varphi_{o}(v)=\frac{\varphi(v)-\varphi(-v)}{2}, \varphi_{e}(v)=\frac{\varphi(v)+\varphi(-v)}{2}, v \in V
$$

respectively. Clearly, $\varphi(v)=\varphi_{e}(v)+\varphi_{o}(v)$ for all $v \in V$.

It is easy to prove that $\varphi_{o}$ and $\varphi_{e}$ satisfies the functional equation (3). By Theorems 8 and 9 , we conclude that $\varphi_{o}$ and $\varphi_{e}$ are additive and quadratic, respectively. Then, there exist a symmetric biadditive mapping $Q: V \times V \longrightarrow W$ which satisfies $\varphi_{e}(v)=Q(v, v)$ and an additive mapping $A: V \longrightarrow W$ which satisfies $\varphi_{o}(v)=A(v)$ for all $v \in V$. Hence, $\varphi(v)=Q(v, v)+A(v)$ for all $v \in V$.

Conversely, suppose that there exists a symmetric biadditive mapping $Q: V \times V \longrightarrow W$ and an additive mapping $A: V \longrightarrow W$ and satisfies $\varphi(v)=Q(v, v)+A(v)$ for all $v \in V$. It is easy to prove that the mappings $v \mapsto Q(v, v)$ and $A: V \longrightarrow W$ satisfy the functional equation (3). Hence, the mapping $\varphi: V \longrightarrow W$ satisfies the functional equation (3).
For our notational convenience, we can define a mapping $\varphi: V \longrightarrow W$ by

$$
\begin{aligned}
D \varphi\left(v_{1}, v_{2}, \cdots, v_{m}\right)= & \varphi\left(\sum_{i=1}^{m} a^{i} v_{i}\right)+\sum_{i=1}^{m} \varphi\left(-a^{i} \mathrm{v}_{i}+\sum_{j=1 ; i \neq j}^{m} a^{j} v_{j}\right) \\
& -(m-3) \sum_{i=1}^{m} \varphi\left(a^{i} v_{i}+a^{j} v_{j}\right) \\
& +\left(m^{2}-5 m+2\right) \sum_{i=1}^{m} a^{2 i}\left[\frac{\varphi\left(v_{i}\right)+\varphi\left(-v_{i}\right)}{2}\right] \\
& +\left(m^{2}-5 m+4\right) \sum_{i=1}^{m} a^{i}\left[\frac{\varphi\left(v_{i}\right)-\varphi\left(-v_{i}\right)}{2}\right],
\end{aligned}
$$

for all $v_{1}, v_{2}, \cdots, v_{m} \in V$.

In the following sections, we consider $V$ is a linear space, $\left(V, I_{\mu^{\prime}, v^{\prime}}, Y\right)$ is an intuitionistic random normed space and $\left(W, I_{\mu, v}, Y\right)$ is a complete intuitionistic random normed space.

\section{Stability Results for Even Case}

Theorem 11. Let $\alpha, \beta: V^{m} \longrightarrow D^{+}$, where $\alpha\left(v_{1}, v_{2}, \cdots, v_{m}\right)$ is denoted by $\alpha_{v_{1}, v_{2}, \cdots, v_{m}}, \beta\left(v_{1}, v_{2}, \cdots, v_{m}\right)$ is denoted by $\beta_{v_{1}, v_{2}, \cdots, v_{m}}$ and $\alpha_{v_{1}, v_{2}, \cdots, v_{m}}(\varepsilon), \beta_{v_{1}, v_{2}, \cdots, v_{m}}(\varepsilon)$ is denoted by $\Theta_{\alpha, \beta}\left(v_{1}, v_{2}, \cdots\right.$, $\left.v_{m}, \varepsilon\right)$, be a mapping such that

$$
\lim _{w \longrightarrow \infty} \Theta_{\alpha, \beta}\left(a^{w} v_{1}, a^{w} v_{2}, \cdots, a^{w} v_{m}, a^{2 w} \varepsilon\right)=1_{L^{*}},
$$

for all $v_{1}, v_{2}, \cdots, v_{m} \in V$ and all $\varepsilon>0$, and

$$
\lim _{w \longrightarrow \infty} \Phi_{i=1}^{\infty}\left(\Theta_{\alpha, \beta}\left(a^{w+i-1} v, 0, \cdots, 0, a^{2 w+i} \varepsilon\right)\right)=1_{L^{*}},
$$

for all $v \in V$ and all $\varepsilon>0$. If an even mapping $\varphi: V \longrightarrow W$ with $\varphi(0)=0$ satisfies

$$
I_{\mu, v}\left(D \varphi\left(v_{1}, v_{2}, \cdots, v_{m}\right), \varepsilon\right) \geq_{L^{*}} \Theta_{\alpha, \beta}\left(v_{1}, v_{2}, \cdots, v_{m}, \varepsilon\right),
$$

for all $v_{1}, v_{2}, \cdots, v_{m} \in V$ and all $\varepsilon>0$, then there exists $a$ unique quadratic mapping $Q_{2}: V \longrightarrow W$ such that

$$
\begin{aligned}
& I_{\mu, v}\left(\varphi(v)-Q_{2}(v), \varepsilon\right) \\
& \quad \geq_{L^{*}} \Phi_{i=1}^{\infty}\left(\Theta_{\alpha, \beta}\left(a^{i-1} v, 0, \cdots, 0, a^{i}\left(m^{2}-5 m+2\right) \varepsilon\right)\right),
\end{aligned}
$$

for all $v \in V$ and all $\varepsilon>0$.

Proof. Replacing $\left(v_{1}, v_{2}, \cdots, v_{m}\right)$ by $(v, 0, \cdots, 0)$ in (29), we have

$$
\begin{aligned}
& I_{\mu, v}\left(\left(m^{2}-5 m+2\right) \varphi(a v)-\left(m^{2}-5 m+2\right) a^{2} \varphi(v), \varepsilon\right) \\
& \quad \geq_{L^{*}} \Theta_{\alpha, \beta}(v, 0, \cdots, 0, \varepsilon),
\end{aligned}
$$


for all $v \in V$ and all $\varepsilon>0$. From inequality (31), we get

$$
\begin{gathered}
I_{\mu, v}\left(\frac{\varphi(a v)}{a^{2}}-\varphi(v), \varepsilon\right) \geq_{L^{*}} \Theta_{\alpha, \beta}\left(v, 0, \cdots, 0, a^{2}\left(m^{2}-5 m+2\right) \varepsilon\right), \\
v \in V, \varepsilon>0 .
\end{gathered}
$$

Interchanging $v$ with $a v$ in (32), we obtain

$$
\begin{aligned}
& I_{\mu, v}\left(\frac{\varphi\left(a^{2} v\right)}{a^{4}}-\frac{\varphi(a v)}{a^{2}}, \varepsilon\right) \\
& \quad \geq_{L^{*}} \Theta_{\alpha, \beta}\left(a v, 0, \cdots, 0, a^{2(2)}\left(m^{2}-5 m+2\right) \varepsilon\right), v \in V, \varepsilon>0 .
\end{aligned}
$$

Replacing $v$ by $a^{l-1} v$ and divide by $a^{2 l}$ in (33), we conclude that

$$
\begin{aligned}
& I_{\mu, v}\left(\frac{\varphi\left(a^{l+1} v\right)}{a^{2(l+1)}}-\frac{\varphi\left(a^{l} v\right)}{a^{2 l}}, \varepsilon\right) \\
& \quad \geq_{L^{*}} \Theta_{\alpha, \beta}\left(a^{l} v, 0, \cdots, 0, a^{2(l+1)}\left(m^{2}-5 m+2\right) \varepsilon\right),
\end{aligned}
$$

for all $v \in V$ and all $\varepsilon>0$. Thus,

$$
\begin{aligned}
& I_{\mu, v}\left(\frac{\varphi\left(a^{w} v\right)}{a^{2 w}}-\varphi(v), \varepsilon\right) \\
& \quad \geq_{L^{*}} \Phi_{l=0}^{w-1}\left(I_{\mu, v}\left(\frac{\varphi\left(a^{l+1} v\right)}{a^{2(l+1)}}-\frac{\varphi\left(a^{l} v\right)}{a^{2 l}}, \sum_{l=0}^{w-1} \frac{\varepsilon}{a^{l+1}}\right)\right),
\end{aligned}
$$

for all $v \in V$ and all $\varepsilon>0$. To prove the convergence of the sequence $\left\{\varphi\left(a^{w} v\right) / a^{2 w}\right\}$, replacing $v$ by $a^{k} v$ in (35), we obtain

$$
\begin{aligned}
& I_{\mu, v}\left(\frac{\varphi\left(a^{w+k} v\right)}{a^{2(w+k)}}-\frac{\varphi\left(a^{k} v\right)}{a^{2(k)}}, \varepsilon\right) \\
& \quad \geq_{L^{*}} \Phi_{l=k}^{w+k-1}\left(I_{\mu, v}\left(\frac{\varphi\left(a^{l+1} v\right)}{a^{2(l+1)}}-\frac{\varphi\left(a^{l} v\right)}{a^{2(l)}}, \sum_{l=k}^{w+k-1} \frac{\varepsilon}{a^{l+1}}\right)\right),
\end{aligned}
$$

for all $v \in V$ and all $\varepsilon>0$ and all $k, w \geq 0$. Since the R.H.S of the inequality (36) tends to $1_{L^{*}}$ as $w, k \longrightarrow \infty$, the sequence $\left\{\varphi\left(a^{w} v\right) / a^{2 w}\right\}$ is a Cauchy sequence in $\left(W, I_{\mu, v}, Y\right)$. Since $\left(W, I_{\mu, v}, Y\right)$ is a complete IRN-space, this sequence converges to some point $Q_{2}(v) \in W$. So one can define the mapping $Q_{2}: V \longrightarrow W$ by

$$
Q_{2}(v)=\lim _{w \longrightarrow \infty} \frac{\varphi\left(a^{w} v\right)}{a^{2 w}},
$$

for all $v \in V$. Letting $k=0$ in (36), we obtain

$$
\begin{aligned}
& I_{\mu, v}\left(\frac{\varphi\left(a^{w} v\right)}{a^{2 w}}-\varphi(v), \varepsilon\right) \\
& \quad \geq_{L^{*}} \Phi_{l=0}^{w-1}\left(I_{\mu, v}\left(\frac{\varphi\left(a^{l+1} v\right)}{a^{2(l+1)}}-\frac{\varphi\left(a^{l} v\right)}{a^{2(l)}}, \sum_{l=0}^{w-1} \frac{\varepsilon}{a^{l+1}}\right)\right),
\end{aligned}
$$

for all $v \in V$ and all $\varepsilon>0$. Taking the limit $w \longrightarrow \infty$ in (38), we get

$$
\begin{aligned}
& I_{\mu, v}\left(\varphi(v)-Q_{2}(v), \varepsilon\right) \\
& \quad \geq_{L^{*}} \Phi_{l=1}^{\infty}\left(\Theta_{\alpha, \beta}\left(a^{l-1} v, 0, \cdots, 0, a^{l}\left(m^{2}-5 m+2\right) \varepsilon\right)\right),
\end{aligned}
$$

for all $v \in V$ and all $\varepsilon>0$.

Next, we prove that the function $Q_{2}$ is quadratic. Replacing $\left(v_{1}, v_{2}, \cdots, v_{m}\right)$ by $\left(a^{w} v_{1}, a^{w} v_{2}, \cdots, a^{w} v_{m}\right)$ in (29), we obtain

$$
\begin{gathered}
I_{\mu, v}\left(\frac{1}{a^{2 w}} D \varphi\left(a^{w} v_{1}, a^{w} v_{2}, \cdots, a^{w} v_{m}\right), \varepsilon\right) \\
\quad \geq_{L^{*}} \Theta_{\alpha, \beta}\left(a^{w} v_{1}, a^{w} v_{2}, \cdots, a^{w} v_{m}, a^{2 w} \varepsilon\right),
\end{gathered}
$$

for all $v_{1}, v_{2}, \cdots, v_{m} \in V$ and all $\varepsilon>0$. Taking the limit as $w \longrightarrow \infty$, we find that $I_{\mu, v}\left(D Q_{2}\left(v_{1}, v_{2}, \cdots, v_{m}\right), \varepsilon\right)=1_{L^{*}}$ for all $v_{1}, v_{2}, \cdots, v_{m} \in V$ and all $\varepsilon>0$, which implies $D Q_{2}\left(v_{1}\right.$, $\left.v_{2}, \cdots, v_{m}\right)=0$. Thus, the function $Q_{2}$ satisfies the functional equation (3). Hence, $Q_{2}: V \longrightarrow W$ is a quadratic mapping. Passing to the limit as $w \longrightarrow \infty$ in (35), we have (30).

Finally, to show the uniqueness of $Q_{2}$ subject to (30), consider that there exists an another quadratic function $Q_{2}^{\prime}$ which satisfies the inequality (30). Clearly, $Q_{2}\left(a^{w} v\right)=a^{2 w} Q_{2}(v)$ and $Q_{2}^{\prime}\left(a^{w} v\right)=a^{2 w} Q_{2}^{\prime}(v)$ for all $v \in V$ and $w \in \mathbb{N}$, from (30) and (28) that

$$
\begin{gathered}
I_{\mu, v}\left(Q_{2}(v)-Q_{2}^{\prime}(v), \varepsilon\right) \geq_{L^{*}} I_{\mu, v}\left(Q_{2}\left(a^{w} v\right)-Q_{2}^{\prime}\left(a^{w} v\right), a^{2 w} \varepsilon\right) \\
\geq_{L^{*}} \Phi\left(I_{\mu, v}\left(Q_{2}\left(a^{w} v\right)-\varphi\left(a^{w} v\right), \frac{a^{2 w}}{2} \varepsilon\right),\right. \\
\left.I_{\mu, v}\left(\varphi\left(a^{w} v\right)-Q_{2}^{\prime}\left(a^{w} v\right), \frac{a^{2 w}}{2} \varepsilon\right)\right) \\
\geq_{L^{*}} \Phi\left(\Phi_{i=1}^{\infty}\left(\Theta_{\alpha, \beta}\left(a^{w+i-1} v, 0, \cdots, 0, a^{2 w+i}\left(m^{2}-5 m+2\right) \frac{\varepsilon}{2}\right)\right),\right. \\
\left.\Phi_{i=1}^{\infty}\left(\Theta_{\alpha, \beta}\left(a^{w+i-1} v, 0, \cdots, 0, a^{2 w+i}\left(m^{2}-5 m+2\right) \frac{\varepsilon}{2}\right)\right)\right),
\end{gathered}
$$

for all $v \in V$ and all $\varepsilon>0$. By taking $w \longrightarrow \infty$ in (41), we show the uniqueness of $Q_{2}$. This ends the proof of the uniqueness, as desired. 
Corollary 12. If an even mapping $\varphi: V \longrightarrow W$ satisfies

$$
I_{\mu, v}\left(D \varphi\left(v_{1}, v_{2}, \cdots, v_{m}, \varepsilon\right) \geq_{L^{*}} I_{\mu^{\prime}, v^{\prime}}\left(\sum_{i=1}^{m} v_{i}, \varepsilon\right)\right.
$$

for all $v_{1}, v_{2}, \cdots, v_{m} \in V$ and all $\varepsilon>0$, and

$$
\lim _{w \longrightarrow \infty} \Phi_{i=1}^{\infty}\left(I_{\mu^{\prime}, v^{\prime}}\left(a^{w+i-1} v, a^{2 w+i}\left(m^{2}-5 m+2\right) \varepsilon\right)\right)=1_{L^{*}},
$$

for all $v \in V$ and all $\varepsilon>0$, then there exists a unique quadratic mapping $Q_{2}: V \longrightarrow W$ such that

$$
I_{\mu, v}\left(\varphi(v)-Q_{2}(v), \varepsilon\right) \geq_{L^{*}} \Phi_{i=1}^{\infty}\left(I_{\mu^{\prime}, v^{\prime}}\left(a^{i-1} v, a^{i}\left(m^{2}-5 m+2\right) \varepsilon\right)\right),
$$

for all $v \in V$ and all $\varepsilon>0$.

Proof. By taking $\Theta_{\alpha, \beta}\left(v_{1}, v_{2}, \cdots, v_{m}, \varepsilon\right)=I_{\mu^{\prime}, v^{\prime}}\left(\sum_{i=1}^{m} v_{i}, \varepsilon\right)$ in Theorem 11, we obtain our desired result.

\section{Stability Results for Odd Case}

Theorem 13. Let $\alpha, \beta: V^{m} \longrightarrow D^{+}$, where $\alpha\left(v_{1}, v_{2}, \cdots, v_{m}\right)$ is denoted by $\alpha_{v_{1}, v_{2}, \cdots, v_{m}}, \beta\left(v_{1}, v_{2}, \cdots, v_{m}\right)$ is denoted by $\beta_{v_{1}, v_{2}, \cdots, v_{m}}$ and $\alpha_{v_{1}, v_{2}, \cdots, v_{m}}(\varepsilon), \beta_{v_{1}, v_{2}, \cdots, v_{m}}(\varepsilon)$ is denoted by $\Theta_{\alpha, \beta}\left(v_{1}, v_{2}, \cdots\right.$, $\left.v_{m}, \varepsilon\right)$, be a mapping such that

$$
\lim _{w \longrightarrow \infty} \Theta_{\alpha, \beta}\left(a^{w} v_{1}, a^{w} v_{2}, \cdots, a^{w} v_{m}, a^{w} \varepsilon\right)=1_{L^{*}},
$$

for all $v_{1}, v_{2}, \cdots, v_{m} \in V$ and all $\varepsilon>0$, and

$$
\lim _{w \longrightarrow \infty} \Phi_{i=1}^{\infty}\left(\Theta_{\alpha, \beta}\left(a^{w+i-1} v, 0, \cdots, 0, a^{w} \varepsilon\right)\right)=1_{L^{*}},
$$

for all $v \in V$ and all $\varepsilon>0$. If an odd mapping $\varphi: V \longrightarrow W$ with $\varphi(0)=0$ satisfies

$$
I_{\mu, v}\left(D \varphi\left(v_{1}, v_{2}, \cdots, v_{m}\right), \varepsilon\right) \geq_{L^{*}} \Theta_{\alpha, \beta}\left(v_{1}, v_{2}, \cdots, v_{m}, \varepsilon\right),
$$

for all $v_{1}, v_{2}, \cdots, v_{m} \in V$ and all $\varepsilon>0$, then there exist a unique additive mapping $A_{1}: V \longrightarrow W$ such that

$$
\begin{aligned}
& I_{\mu, v}\left(\varphi(v)-A_{1}(v), \varepsilon\right) \\
& \quad \geq_{L^{*}} \Phi_{i=1}^{\infty}\left(\Theta_{\alpha, \beta}\left(a^{i-1} v, 0, \cdots, 0,\left(m^{2}-5 m+4\right) \varepsilon\right)\right),
\end{aligned}
$$

for all $v \in V$ and all $\varepsilon>0$.

Proof. Replacing $\left(v_{1}, v_{2}, \cdots, v_{m}\right)$ by $(v, 0, \cdots, 0)$ in (47), we obtain

$$
\begin{aligned}
& I_{\mu, v}\left(\left(m^{2}-5 m+4\right) \varphi(a v)-\left(m^{2}-5 m+4\right) a \varphi(v), \varepsilon\right) \\
& \quad \geq_{L^{*}} \Theta_{\alpha, \beta}(v, 0, \cdots, 0, \varepsilon),
\end{aligned}
$$

for all $v \in V$ and all $\varepsilon>0$. From inequality (49), we get

$$
I_{\mu, v}\left(\frac{\varphi(a v)}{a}-\varphi(v), \varepsilon\right) \geq_{L^{*}} \Theta_{\alpha, \beta}\left(v, 0, \cdots, 0, a\left(m^{2}-5 m+4\right) \varepsilon\right)
$$

for all $v \in V$ and all $\varepsilon>0$. Replacing $v$ by a $v$ in the above inequality (50), we have

$$
\begin{aligned}
I_{\mu, v} & \left(\frac{\varphi\left(a^{2} v\right)}{a^{2}}-\frac{\varphi(a v)}{a}, \varepsilon\right) \\
& \geq_{L^{*}} \Theta_{\alpha, \beta}\left(a v, 0, \cdots, 0, a^{2}\left(m^{2}-5 m+4\right) \varepsilon\right),
\end{aligned}
$$

for all $v \in V$ and all $\varepsilon>0$. Replacing $v$ by $a^{l-1} v$ in (51), we conclude that

$$
\begin{aligned}
& I_{\mu, v}\left(\frac{\varphi\left(a^{l+1} v\right)}{a^{l+1}}-\frac{\varphi\left(a^{l} v\right)}{a^{l}}, \varepsilon\right) \\
& \quad \geq_{L^{*}} \Theta_{\alpha, \beta}\left(a^{l} v, 0, \cdots, 0, a^{(l+1)}\left(m^{2}-5 m+4\right) \varepsilon\right),
\end{aligned}
$$

for all $v \in V$ and all $\varepsilon>0$. Thus,

$$
\begin{aligned}
& I_{\mu, v}\left(\frac{\varphi\left(a^{w} v\right)}{a^{w}}-\varphi(v), \varepsilon\right) \\
& \quad \geq_{L^{*}} \Phi_{l=0}^{w-1}\left(I_{\mu, v}\left(\frac{\varphi\left(a^{l+1} v\right)}{a^{(l+1)}}-\frac{\varphi\left(a^{l} v\right)}{a^{l}}, \sum_{l=0}^{w-1} \frac{\varepsilon}{a^{l+1}}\right)\right),
\end{aligned}
$$

for all $v \in V$ and all $\varepsilon>0$. To prove the convergence of the sequence $\left\{\varphi\left(a^{w} v\right) / a^{w}\right\}$, replacing $v$ by $a^{k} v$ in (53), we obtain

$$
\begin{aligned}
& I_{\mu, v}\left(\frac{\varphi\left(a^{w+k} v\right)}{a^{(w+k)}}-\frac{\varphi\left(a^{k} v\right)}{a^{(k)}}, \varepsilon\right) \\
& \quad \geq_{L^{*}} \Phi_{l=k}^{w+k-1}\left(I_{\mu, v}\left(\frac{\varphi\left(a^{l+1} v\right)}{a^{(l+1)}}-\frac{\varphi\left(a^{l} v\right)}{a^{(l)}}, \sum_{l=k}^{w+k-1} \frac{\varepsilon}{a^{l+1}}\right)\right),
\end{aligned}
$$

for all $v \in V$ and all $\varepsilon>0$ and all $k, w \geq 0$. Since the R.H.S of the inequality (54) tends to $1_{L^{*}}$ as $w, k \longrightarrow \infty$, the sequence $\left\{\varphi\left(a^{w} v\right) / a^{w}\right\}$ is a Cauchy sequence in $\left(W, I_{\mu, v}, Y\right)$. Since $\left(W, I_{\mu, v}, Y\right)$ is a complete IRN-space, this sequence converges to some point $A_{1}(v) \in W$. So one can define the mapping $A_{1}: V \longrightarrow W$ by

$$
A_{1}(v)=\lim _{w \longrightarrow \infty} \frac{\varphi\left(a^{w} v\right)}{a^{w}},
$$


for all $v \in V$. Letting $k=0$ in (54), we obtain

$$
\begin{aligned}
& I_{\mu, v}\left(\frac{\varphi\left(a^{w} v\right)}{a^{w}}-\varphi(v), \varepsilon\right) \\
& \quad \geq_{L^{*}} \Phi_{l=0}^{w-1}\left(I_{\mu, v}\left(\frac{\varphi\left(a^{l+1} v\right)}{a^{(l+1)}}-\frac{\varphi\left(a^{l} v\right)}{a^{(l)}}, \sum_{l=0}^{w-1} \frac{\varepsilon}{a^{l+1}}\right)\right),
\end{aligned}
$$

for all $v \in V$ and all $\varepsilon>0$. Taking the limit as $w \longrightarrow \infty$ in (56), we get

$$
\begin{aligned}
& I_{\mu, v}\left(\varphi(v)-A_{1}(v), \varepsilon\right) \\
& \quad \geq_{L^{*}} \Phi_{l=1}^{\infty}\left(\Theta_{\alpha, \beta}\left(a^{l-1} v, 0, \cdots, 0,\left(m^{2}-5 m+4\right) \varepsilon\right)\right),
\end{aligned}
$$

for all $v \in V$ and all $\varepsilon>0$.

Next, we want to prove that the function $A_{1}$ is additive. Replacing $\left(v_{1}, v_{2}, \cdots, v_{m}\right)$ by $\left(a^{w} v_{1}, a^{w} v_{2}, \cdots, a^{w} v_{m}\right)$ in (47), we obtain

$$
\begin{array}{r}
I_{\mu, v}\left(\frac{1}{a^{w}} D \varphi\left(a^{w} v_{1}, a^{w} v_{2}, \cdots, a^{w} v_{m}\right), \varepsilon\right) \\
\quad \geq_{L^{*}} \Theta_{\alpha, \beta}\left(a^{w} v_{1}, a^{w} v_{2}, \cdots, a^{w} v_{m}, a^{w} \varepsilon\right),
\end{array}
$$

for all $v_{1}, v_{2}, \cdots, v_{m} \in V$ and all $\varepsilon>0$. Taking the limit as $w \longrightarrow \infty$, we find that $I_{\mu, v}\left(D A_{1}\left(v_{1}, v_{2}, \cdots, v_{m}\right), \varepsilon\right)=1_{L^{*}}$ for all $v_{1}, v_{2}, \cdots, v_{m} \in V$ and all $\varepsilon>0$, which implies $D A_{1}\left(v_{1}\right.$, $\left.v_{2}, \cdots, v_{m}\right)=0$. Thus, $A_{1}$ satisfies the functional equation (3). Hence, the function $A_{1}: V \longrightarrow W$ is additive. Passing to the limit as $w \longrightarrow \infty$ in (53), we have (48).

Finally, to show the uniqueness of the additive function $A_{1}$ subject to (48), consider that there exists another additive function $A_{1}^{\prime}$ which satisfies the inequality (48). Evidently, $A_{1}$ $\left(a^{w} v\right)=a^{w} A_{1}(v)$ and $A_{1}^{\prime}\left(a^{w} v\right)=a^{w} A_{1}^{\prime}(v)$ for all $v \in V$ and $w$ $\in \mathbb{N}$, from (48) and (46) that

$$
\begin{gathered}
I_{\mu, v}\left(A_{1}(v)-A_{1}^{\prime}(v), \varepsilon\right) \geq_{L^{*}} I_{\mu, v}\left(A_{1}\left(a^{w} v\right)-A_{1}^{\prime}\left(a^{w} v\right), a^{w} \varepsilon\right) \\
\geq_{L^{*}} \Phi\left(I_{\mu, v}\left(A_{1}\left(a^{w} v\right)-\varphi\left(a^{w} v\right), \frac{a^{w}}{2} \varepsilon\right),\right. \\
\left.I_{\mu, v}\left(\varphi\left(a^{w} v\right)-A_{1}^{\prime}\left(a^{w} v\right), \frac{a^{w}}{2} \varepsilon\right)\right) \\
\geq_{L^{*}} \Phi\left(\Phi_{i=1}^{\infty}\left(\Theta_{\alpha, \beta}\left(a^{w+i-1} v, 0, \cdots, 0, a^{w}\left(m^{2}-5 m+4\right) \frac{\varepsilon}{2}\right)\right),\right. \\
\left.\Phi_{i=1}^{\infty}\left(\Theta_{\alpha, \beta}\left(a^{w+i-1} v, 0, \cdots, 0, a^{w}\left(m^{2}-5 m+4\right) \frac{\varepsilon}{2}\right)\right)\right),
\end{gathered}
$$

for all $v \in V$ and all $\varepsilon>0$. By taking the limit $w \longrightarrow \infty$ in (59), we show the uniqueness of $A_{1}$.

Corollary 14. If an odd mapping $\varphi: V \longrightarrow W$ satisfies

$$
I_{\mu, v}\left(D \varphi\left(v_{1}, v_{2}, \cdots, v_{m}, \varepsilon\right) \geq_{L^{*}} I_{\mu^{\prime}, v^{\prime}}\left(\sum_{i=1}^{m} v_{i}, \varepsilon\right),\right.
$$

for all $v_{1}, v_{2}, \cdots, v_{m} \in V$ and all $\varepsilon>0$, and

$$
\lim _{w \longrightarrow \infty} \Phi_{i=1}^{\infty}\left(I_{\mu^{\prime}, v^{\prime}}\left(a^{w+i-1} v, a^{w}\left(m^{2}-5 m+4\right) \varepsilon\right)\right)=1_{L^{*}},
$$

for all $v \in V$ and all $\varepsilon>0$. Then, there exists a unique additive mapping $A_{1}: V \longrightarrow W$ such that

$$
I_{\mu, v}\left(\varphi(v)-A_{1}(v), \varepsilon\right) \geq_{L^{*}} \Phi_{i=1}^{\infty}\left(I_{\mu^{\prime}, v^{\prime}}\left(a^{i-1} v,\left(m^{2}-5 m+4\right) \varepsilon\right)\right)
$$

for all $v \in V$ and all $\varepsilon>0$.

Proof. By taking $\Theta_{\alpha, \beta}\left(v_{1}, v_{2}, \cdots, v_{m}, \varepsilon\right)=I_{\mu^{\prime}, v^{\prime}}\left(\sum_{i=1}^{m} v_{i}, \varepsilon\right)$ in Theorem 13, we obtain our desired result.

\section{Stability Results for Mixed Case}

Theorem 15. Let $\alpha, \beta: V^{m} \longrightarrow D^{+}$be mappings satisfying (27), (28), (45), and (46) for all $v_{1}, v_{2}, \cdots, v_{m}, v \in V$ and all $\varepsilon$ $>0$. If a mapping $\varphi: V \longrightarrow W$ with $\varphi(0)=0$ satisfies (29) for all $v_{1}, v_{2}, \cdots, v_{m} \in V$ and all $\varepsilon>0$, then there exist a unique quadratic mapping $Q_{2}: V \longrightarrow W$ and a unique additive mapping $A_{1}: V \longrightarrow W$ satisfying (3) and

$$
\begin{aligned}
I_{\mu, v}( & \left.\varphi(v)-Q_{2}(v)-A_{1}(v), \varepsilon\right) \\
\geq_{L^{*}} & \Phi\left(\Phi\left(\Phi_{i=1}^{\infty}\left(a^{i-1} v, 0, \cdots, 0, a^{i}\left(m^{2}-5 m+2\right) \varepsilon\right)\right),\right. \\
& \left.\left.\Phi_{i=1}^{\infty}\left(-a^{i-1} v, 0, \cdots, 0, a^{i}\left(m^{2}-5 m+2\right) \varepsilon\right)\right)\right) \\
& \left(\Phi\left(\Phi_{i=1}^{\infty}\left(a^{i-1} v, 0, \cdots, 0,\left(m^{2}-5 m+4\right) \varepsilon\right)\right),\right. \\
& \left.\left.\Phi_{i=1}^{\infty}\left(-a^{i-1} v, 0, \cdots, 0,\left(m^{2}-5 m+4\right) \varepsilon\right)\right)\right),
\end{aligned}
$$

for all $v \in V$.

Proof. Let $\varphi_{e}(v)=\varphi(v)+\varphi(-v) / 2$ for all $v \in V$. Thus, $\varphi_{e}$ $(0)=0, \varphi_{e}(-v)=\varphi_{e}(v)$ and for all $v_{1}, v_{2}, \cdots, v_{m} \in V$ and all $\varepsilon>0$,

$$
\begin{aligned}
& I_{\mu, v}\left(D \varphi_{e}\left(v_{1}, v_{2}, \cdots, v_{m}\right), \varepsilon\right) \\
& =I_{\mu, v}\left(\frac{D \varphi\left(v_{1}, v_{2}, \cdots, v_{m}\right)+D \varphi\left(-v_{1},-v_{2}, \cdots,-v_{m}\right)}{2}, \varepsilon\right), \\
& \geq_{L^{*}} \Phi\left(I_{\mu, v}\left(D \varphi\left(v_{1}, v_{2}, \cdots, v_{m}\right), \varepsilon\right), I_{\mu, v}\left(D \varphi\left(-v_{1},-v_{2}, \cdots,-v_{m}\right), \varepsilon\right)\right), \\
& \geq_{L^{*}} \Phi\left(\Theta_{\alpha, \beta}\left(v_{1}, v_{2}, \cdots, v_{m}, \varepsilon\right), \Theta_{\alpha, \beta}\left(-v_{1},-v_{2}, \cdots,-v_{m}, \varepsilon\right)\right) .
\end{aligned}
$$

By Theorem 11, there exists a quadratic mapping $Q_{2}: V \longrightarrow W$ such that

$$
\begin{gathered}
I_{\mu, v}\left(\varphi_{e}(v)-Q_{2}(v), \varepsilon\right) \\
\geq_{L^{*}} \Phi\left(\Phi_{i=1}^{\infty}\left(a^{i-1} v, 0, \cdots, 0, a^{i}\left(m^{2}-5 m+2\right) \varepsilon\right),\right. \\
\left.\Phi_{i=1}^{\infty}\left(-a^{i-1} v, 0, \cdots, 0, a^{i}\left(m^{2}-5 m+2\right) \varepsilon\right)\right),
\end{gathered}
$$

for all $v \in V$ and all $\varepsilon>0$. 
On the other hand, let $\varphi_{o}(v)=\varphi(v)-\varphi(-v) / 2$ for all $v \in V$. Then $\varphi_{o}(0)=0, \varphi_{o}(-v)=-\varphi_{o}(v)$. By Theorem 13, there exists a additive mapping $A_{1}: V \longrightarrow W$ satisfies

$$
\begin{gathered}
I_{\mu, v}\left(\varphi_{0}(v)-A_{1}(v), \varepsilon\right) \\
\geq_{L^{*}} \Phi\left(\Phi_{i=1}^{\infty}\left(a^{i-1} v, 0, \cdots, 0,\left(m^{2}-5 m+4\right) \varepsilon\right),\right. \\
\left.\Phi_{i=1}^{\infty}\left(-a^{i-1} v, 0, \cdots, 0,\left(m^{2}-5 m+4\right) \varepsilon\right)\right),
\end{gathered}
$$

for all $v \in V$ and all $\varepsilon>0$. From inequalities (65) and (66), we obtain our desired result (64).

Corollary 16. If a mapping $\varphi: V \longrightarrow W$ satisfies

$$
I_{\mu, v}\left(D \varphi\left(v_{1}, v_{2}, \cdots, v_{m}, \varepsilon\right) \geq_{L^{*}} I_{\mu^{\prime}, v^{\prime}}\left(\sum_{i=1}^{m} v_{i}, \varepsilon\right)\right.
$$

for all $v_{1}, v_{2}, \cdots, v_{m} \in V$ and all $\varepsilon>0$, and

$$
\begin{gathered}
\lim _{w \longrightarrow \infty} \Phi_{i=1}^{\infty}\left(I_{\mu^{\prime}, v^{\prime}}\left(a^{w+i-1} v, a^{2 w+i}\left(m^{2}-5 m+2\right) \varepsilon\right)\right)=1_{L^{*}}, \\
\lim _{w \longrightarrow \infty} \Phi_{i=1}^{\infty}\left(I_{\mu^{\prime}, v^{\prime}}\left(a^{w+i-1} v, a^{w}\left(m^{2}-5 m+4\right) \varepsilon\right)\right)=1_{L^{*}},
\end{gathered}
$$

for all $v \in V$ and all $\varepsilon>0$. Then, there exists a unique quadratic mapping $Q_{2}: V \longrightarrow W$ and a unique additive mapping $A_{1}: V \longrightarrow W$ such that

$$
\begin{gathered}
I_{\mu, v}\left(\varphi(v)-Q_{2}(v)-A_{1}(v), \varepsilon\right) \\
\geq_{L^{*}} \Phi\left(\Phi\left(\Phi_{i=1}^{\infty}\left(a^{i-1} v, a^{i}\left(m^{2}-5 m+2\right) \varepsilon\right)\right),\right. \\
\left.\left.\Phi_{i=1}^{\infty}\left(-a^{i-1} v, a^{i}\left(m^{2}-5 m+2\right) \varepsilon\right)\right)\right) \\
\cdot\left(\Phi\left(\Phi_{i=1}^{\infty}\left(a^{i-1} v,\left(m^{2}-5 m+4\right) \varepsilon\right)\right),\right. \\
\left.\left.\Phi_{i=1}^{\infty}\left(-a^{i-1} v,\left(m^{2}-5 m+4\right) \varepsilon\right)\right)\right),
\end{gathered}
$$

for all $v \in V$ and all $\varepsilon>0$.

\section{Conclusion}

In this paper, we introduced a new mixed type of additivequadratic functional equation, and we applied the Hyers direct technique to investigate the Hyers-Ulam stability of the mixed type of additive-quadratic functional equation. Moreover, we have derived its general solution. The main objective of this work has been discussed: In Section 4, we have proved its Ulam-Hyers stability for the even case; in Section 5, examined Ulam-Hyers stability for odd case, and in Section 6, investigated Ulam-Hyers stability for the mixed cases, respectively, in intuitionistic random normed space.

\section{Data Availability}

No data were used in this study.

\section{Conflicts of Interest}

The authors declare that they have no conflicts of interest.

\section{Acknowledgments}

This research was funded by the Deanship of Scientific Research at Princess Nourah Bint Abdulrahman University through the Fast-track Research Funding Program.

\section{References}

[1] S. M. Ulam, "A collection of mathematical problems," in Interscience Tracts in Pure and Applied Mathematics, no. 8, 1960Interscience Publishers, New York, 1960.

[2] D. H. Hyers, "On the stability of the linear functional equation," Proceedings of the National Academy of Sciences of the United States of America, vol. 27, no. 4, pp. 222-224, 1941.

[3] T. Aoki, "On the stability of the linear transformation in Banach spaces," Journal of the Mathematical Society of Japan, vol. 2, pp. 64-66, 1950.

[4] T. M. Rassias, "On the stability of the linear mapping in Banach spaces," Proceedings of the American Mathematical Society, vol. 72, no. 2, pp. 297-300, 1978.

[5] P. Gavruta, "A generalization of the Hyers-Ulam-Rassias stability of approximately additive mappings," Journal of Mathematical Analysis and Applications, vol. 184, no. 3, pp. 431-436, 1994.

[6] Z. Gajda, "On stability of additive mappings," International Journal of Mathematics and Mathematical Sciences, vol. 14, no. 3, 434 pages, 1991.

[7] S.-M. Jung, Hyers-Ulam-Rassias Stability of Functional Equations in Mathematical Analysis, Hadronic Press, Inc., Palm Harbor, FL, 2001.

[8] S.-M. Jung, "On the Hyers-Ulam stability of the functional equations that have the quadratic property," Journal of Mathematical Analysis and Applications, vol. 222, no. 1, pp. 126137, 1998.

[9] H. Khodaei and T. M. Rassias, "Approximately generalized additive functions in several variables," International Journal of Nonlinear Analysis and Applications, vol. 1, pp. 22-41, 2010.

[10] T. M. Rassias, "On the stability of functional equations and a problem of Ulam," Acta Applicandae Mathematicae, vol. 62, no. 1, pp. 23-130, 2000.

[11] T. M. Rassias and P. Šemrl, "On the Hyers-Ulam stability of linear mappings," Journal of Mathematical Analysis and Applications, vol. 173, no. 2, pp. 325-338, 1993.

[12] K. Tamilvanan, J. R. Lee, and C. Park, "Ulam stability of a functional equation deriving from quadratic and additive mappings in random normed spaces," AIMS Mathematics, vol. 6, no. 1, pp. 908-924, 2021.

[13] A. Najati and M. B. Moghimi, "Stability of a functional equation deriving from quadratic and additive functions in quasiBanach spaces," Journal of Mathematical Analysis and Applications, vol. 337, no. 1, pp. 399-415, 2008.

[14] A. Najati and T. M. Rassias, "Stability of a mixed functional equation in several variables on Banach modules," Nonlinear Analysis, vol. 72, no. 3-4, pp. 1755-1767, 2010.

[15] A. Najati and G. Z. Eskandani, "Stability of a mixed additive and cubic functional equation in quasi-Banach spaces," 
Journal of Mathematical Analysis and Applications, vol. 342, no. 2, pp. 1318-1331, 2008.

[16] K.-W. Jun and H.-M. Kim, "Ulam stability problem for a mixed type of cubic and additive functional equation," Bulletin of the Belgian Mathematical Society, vol. 13, no. 2, pp. 271-285, 2006.

[17] H.-M. Kim, "On the stability problem for a mixed type of quartic and quadratic functional equation," Journal of Mathematical Analysis and Applications, vol. 324, no. 1, pp. 358-372, 2006.

[18] N. Alessa, K. Tamilvanan, G. Balasubramanian, and K. Loganathan, "Stability results of the functional equation deriving from quadratic function in random normed spaces," AIMS Mathematics, vol. 6, no. 3, pp. 2385-2397, 2021.

[19] S. O. Kim and K. Tamilvanan, "Fuzzy stability results of generalized quartic functional equations," Mathematics, vol. 9, 2021.

[20] J. R. Lee, J. Kim, and C. Park, "A fixed point approach to the stability of an additive-quadratic-cubic-quartic functional equation," Fixed Point Theory and Applications, vol. 2010, Article ID 185780, 17 pages, 2010.

[21] C. Park, "A fixed point approach to the fuzzy stability of an additive-quadratic-cubic functional equation," Fixed Point Theory and Applications, vol. 2009, Article ID 918785, 24 pages, 2009.

[22] C. Park, "Fuzzy stability of a functional equation associated with inner product spaces," Fuzzy Sets and Systems, vol. 160, no. 11, pp. 1632-1642, 2009.

[23] S. Zhang, J. M. Rassias, and R. Saadati, "Stability of a cubic functional equation in intuitionistic random normed spaces," Applied Mathematics and Mechanics, vol. 31, no. 1, pp. 2126, 2010.

[24] C. Alsina, "On the stability of a functional equation arising in probabilistic normed spaces," in General inequalities, 5 (Oberwolfach, 1986), pp. 263-271, Birkhäuser, Basel, 1985.

[25] M. Eshaghi Gordji, M. B. Ghaemi, and H. Majani, "Generalized Hyers-Ulam-Rassias theorem in Menger probabilistic normed spaces," Discrete Dynamics in Nature and Society, vol. 2010, Article ID 162371, 11 pages, 2010.

[26] M. Gordji, M. B. Ghaemi, H. Majani, and C. Park, "Generalized Ulam-Hyers stability of Jensen functional equation in Šerstnev PN spaces," Journal of Inequalities and Applications, vol. 2010, Article ID 868193, 14 pages, 2010.

[27] D. Mihet and V. Radu, "On the stability of the additive Cauchy functional equation in random normed spaces," Journal of mathematical Analysis and Applications, vol. 343, no. 1, pp. 567-572, 2008.

[28] D. Miheț, R. Saadati, and S. M. Vaezpour, "The stability of the quartic functional equation in random normed spaces," Acta Applicandae Mathematicae, vol. 110, no. 2, pp. 797-803, 2010.

[29] D. Miheț, R. Saadati, and S. M. Vaezpour, "The stability of an additive functional equation in Menger probabilistic $\varphi$ normed spaces," Mathematica Slovaca, vol. 61, no. 5, pp. 817-826, 2011.

[30] R. Saadati, S. M. Vaezpour, and Y. J. Cho, "A note to paper "On the stability of cubic mappings and quartic mappings in random normed Spaces" (Erratum)," Journal of Inequalities and Applications, vol. 2009, 6 pages, 2009.

[31] T. Z. Xu, J. M. Rassias, and W. X. Xu, "Intuitionistic fuzzy stability of a general mixed additive-cubic equation," Journal of Mathematical Physics, vol. 51, no. 6, article 063519, 2010.
[32] T. Z. Xu, J. M. Rassias, and W. X. Xu, "Generalized HyersUlam stability of a general mixed additive-cubic functional equation in quasi-Banach spaces," Acta Mathematica Sinica, English Series, vol. 28, pp. 529-560, 2012.

[33] T. Z. Xu, J. M. Rassias, and W. X. Xu, "On the stability of a general mixed additive-cubic functional equation in random normed spaces," Journal of Inequalities and Applications, vol. 2010, no. 1, 2010.

[34] Y. Guo, M. Chen, X. B. Shu, and F. Xu, "The existence and Hyers-Ulam stability of solution for almost periodical fractional stochastic differential equation with $\mathrm{fBm}$," Stochastic Analysis and Applications, vol. 39, no. 4, pp. 643-666, 2021.

[35] S. Li, L. Shu, X. B. Shu, and F. Xu, "Existence and Hyers-Ulam stability of random impulsive stochastic functional differential equations with finite delays," Stochastics, vol. 91, no. 6, pp. 857-872, 2019.

[36] S. Chang, Y. J. Cho, and S. M. Kang, Nonlinear Operator Theory in Probabilistic Metric Spaces, Nova Science Publishers, Inc., Huntington, NY, 2001.

[37] O. Hadžić and E. Pap, "Fixed point theory in probabilistic metric spaces," in Mathematics and its Applications, vol. 536, Kluwer Academic Publishers, Dordrecht, 2001.

[38] S. Kutukcu, A. Tuna, and A. T. Yakut, "Generalized contraction mapping principle in intuitionistic Menger spaces and application to differential equations," Applied Mathematics and Mechanics, vol. 28, no. 6, pp. 799-809, 2007.

[39] R. Saadati and J. H. Park, "On the intuitionistic fuzzy topological spaces," Chaos, Solitons and Fractals, vol. 27, no. 2, pp. 331-344, 2006.

[40] B. Schweizer and A. Sklar, Probabilistic Metric Spaces, NorthHolland Series in Probability and Applied Mathematics, North-Holland Publishing Co., New York, 1983.

[41] A. N. Šerstnev, "Best-approximation problems in random normed spaces," Doklady Akademii Nauk SSSR, vol. 149, pp. 539-542, 1963.

[42] J. M. Rassias, R. Saadati, G. Sadeghi, and J. Vahidi, "On nonlinear stability in various random normed spaces," Journal of Inequalities and Applications, vol. 2011, no. 1, 2011.

[43] K. T. Atanassov, "Intuitionistic fuzzy sets," Fuzzy Sets and Systems, vol. 20, no. 1, pp. 87-96, 1986.

[44] G. Deschrijver and E. E. Kerre, "On the relationship between some extensions of fuzzy set theory," Fuzzy Sets and Systems, vol. 133, no. 2, pp. 227-235, 2003. 